\title{
La Storia della letteratura italiana come romanzo
}

\author{
Remo Ceserani \\ Università di Bologna \\ remo.ceserani@unibo.it
}

\begin{abstract}
La Storia della letteratura italiana di Francesco De Sanctis viene letta in questa relazione come un romanzo, forse il romanzo di maggior successo dell'Ottocento italiano, dopo I promessi sposi di Alessandro Manzoni. Nell'interpretazione di Ceserani la struttura del libro è basata su tre tipi molto popolari di narrazione praticati in quel secolo: 1) il romanzo storico, in cui realtà e invenzione sono mescolate e scene di movimento di grandi masse si alternano con scene in cui singoli grandi eroi (Dante, Machiavelli) sono celebrati e rappresentati nella loro monumentalità: 2) il romanzo di educazione o Bildungsroman, nel quale tuttavia il protagonista non è un singolo personaggio, che costruisce la sua individualità dalla giovinezza alla maturità, ma è, invece, un intero popolo, che passa attraverso fasi successive: una fase creativa e spontanea nel Trecento, una fase di decadenza nel Cinquecento e Seicento, una nuova partenza e un vero rinascimento nell'età moderna; 3) il dramma borghese e l'opera in musica, con il loro gusto per i contrasti dialettici e i forti conflitti.

Parole chiave: Storia; Letteratura; Romanzo; Bildungsroman; Dramma.
\end{abstract}

\begin{abstract}
Francesco De Sanctis' History of Italian Literature is read in this paper as a novel, probably the most successful novel of the Italian Nineteenth-Century after The betrothed by Alessandro Manzoni. According to Ceserani's interpretation, the structure of the book is based on three popular types of narrations written in that century: 1) the historical novel (or romance), in which reality and invention are combined and scenes of large masses alternate with scenes in which single great heroes (Dante, Machiavelli) are celebrated and represented in their monumentality; 2) the novel of education or Bildungsroman, but in this case the protagonist is not a single character, who constructs his individuality from youth to maturity; instead it is an entire people, who passes through different stages: a very creative and genuine one in the Trecento, an age of decadence during the Cinquecento and Seicento, a new start and a true renaissance in the modern times; 3) the bourgeois drama and the opera, with their penchant for dialectical contrasts and sharp conflicts.
\end{abstract}

Keywords: History; Literature; Novel; Bildungsroman; Drama. 
Mi è capitato più volte di scrivere ${ }^{1}$ che la Storia della letteratura italiana (187071) di Francesco De Sanctis è forse, dopo i Promessi sposi, il più bel romanzo italiano dell'Ottocento. Provo qui ad approfondire questa idea.

Richiamo anzitutto i generi letterari, tutti di forte presenza nella letteratura dell'Ottocento, a cui il libro di De Sanctis si ispira. Essi sono il romanzo storico, il romanzo di formazione e il dramma borghese.

\section{La Storia della letteratura italiana come romanzo storico}

Fra le caratteristiche tipiche del romanzo storico la Storia della letteratura italiana ha preso, anzitutto, quello che ne forma la struttura narrativa più ampia e cioè l'alternanza di scene-affresco di massa con scene in cui su tutto spicca e si staglia l'eroe-individuo. Si possono prendere, come esempi di questa particolare struttura, i modi in cui sono rappresentati Dante e Savonarola. Ecco il primo, in un ritratto che arieggia i personaggi della pittura romantica e anche dell'opera in musica ottocentesca:

Dante accigliato, brusco, tutto di un pezzo, com'è ne' suoi ritratti, ha troppa bile e collera, e non è buono né alla caricatura, né all'ironia. Ma dalla sua fantasia d'artista è uscita una di quelle creazioni, che sono le grandi scoperte nella storia dell'arte, un mondo nuovo: il nero cherubino, che strappa a San Francesco l'anima di Guido da Montefeltro, è il padre di Mefistofele. ${ }^{2}$

Accanto a Dante, altre figure in forte rilievo scultoreo: Brunetto, Pier delle Vigne, Francesca:

Queste grandi figure, là sul loro piedistallo rigide ed epiche come statue, attendono l'artista che le prenda per mano e le gitti nel tumulto della vita e le faccia esseri drammatici. E l'artista non fu un italiano: fu Shakespeare. (SLI, p. 275)

Il collegamento fra i due grandi artisti del canone romantico, Dante e Shakespeare, introduce qui un forte nesso (i formalisti russi avrebbero parlato di elementi tematici «legati»), facendo risaltare la trama narrativa del libro. Non diversamente avviene quando sulla pagina prende posto Savonarola, anche lui con spicco scultoreo e drammatico, anche lui presentato come un elemento fondamentale della trama narrativa che lega fra loro le epoche storiche:

[Savonarola] parve l'ombra scura e vindice del medio evo, che riapparisse d'improvviso nel mondo tra frati e plebe e gitta nel rogo Petrarca, Boccaccio, Pulci, Poliziano, Lorenzo e gli altri peccatori, e rovescia il carro di Bacco e

1. Per esempio Remo Ceserani, Raccontare la letteratura, Torino: Bollati Boringhieri, 1990, p. 19-23.

2. Francesco De Sanctis, Storia della letteratura italiana, a cura di Grazia Melli Fioravanti, introduzione di René Wellek, Milano, Rizzoli BUR, 1983, p. 269. D’ora in avanti indicata nel testo con la sigla SLI e il numero delle pagine. 
Arianna, e ritta sul carro della Morte tende la mano minacciosa e con voce nunzia di sciagure grida agli uomini: — Penitenza! penitenza! — (SLI, p. 473)

A Savonarola si contrappone, nell'immaginaria scena drammatica, e con altrettanto spiccati tratti somatici e di carattere, e in posizione chiave nell'impianto registico, il Segretario fiorentino:

Niccolò Machiavelli, ne' suoi tratti apparenti, è una fisionomia essenzialmente fiorentina ed ha molta somiglianza con Lorenzo de'Medici. Era un piacevolone, che si spassava ben volentieri tra le confraternite e le liete brigate [...] Ma caduti i Medici, ristaurata la repubblica e nominato segretario, ebbe parte principalissima nelle pubbliche faccende. [...] In quegli uffici e in quelle lotte si raffermò la sua tempra e si formò il suo spirito. (SLI, p. 590)

Purtroppo quello spirito, per divenire carattere nazionale ed esempio di riscatto, dovette cedere la scena ad altri personaggi: Giovanni Boccaccio, Ludovico Ariosto, Pietro Aretino, protagonisti (con tratti fra il comico e il disincantato) di un mondo in cui "non è alcuna serietà, non patria, non famiglia». Ecco Aretino, ritratto, come Ariosto, da Tiziano, trasformato da De Sanctis in maschera teatrale:

Ebbe da natura grandi appetiti e forze proporzionate. Vedi il suo ritratto, fatto da Tiziano. Figura di lupo che cerca la preda. L'incisore gli formò la cornice di pelle e zampe di lupo; e la testa del lupo assai simile di struttura sta sopra alla testa dell'uomo. Occhi scintillanti, narici aperte, denti in evidenza per il labbro inferiore abbassato, grossissima la parte posteriore del capo, sede degli appetiti sensuali, verso la quale pare che si gitti la testa, calva nella parte anteriore. (SLI, p. 654)

Come esempio di scene-affresco si può leggere la pagina in cui De Sanctis, riscrivendo un celebre passo di Dino Compagni, descrive la folla dei pellegrini cristiani accorsi a Roma per il Giubileo del 1300: la pagina è costruita con la stessa tecnica narrativa di alcune famose scene corali o di massa di Michelet (o di Balzac o Zola, o Verdi), mescolando gli elementi tratti dalla cronaca e dalle fonti storiche con elementi immaginari e di fantasia:

Tutta la cristianità concorse a Roma, d'ogni età, d'ogni sesso, di ogni ordine e condizione, per ottenere il perdono de peccati e guadagnarsi la salute eterna. Tutti animava lo stesso concetto espresso così variamente in tante prose e poesie, la maledizione del mondo e della carne, la vanità de' beni e delle cure terrestri e la vita cercata al di là della vita. Il nuovo secolo cominciava, consacrando in modo tanto solenne il pensiero comune nella varietà della coltura. I preti e i frati soprastavano nella riverenza pubblica, non solo pel carattere religioso, ma per la dottrina, tenuta loro privilegio, tanto che il Villani loda di scienza Dante, aggiungendo: «benché laico», e i dotti uomini, benché laici, erano detti chierici. Tutta la società italiana, raccolta colà dallo stesso fine, rendeva una viva immagine di quel pensiero comune e di quella varia cultura. Vedevi i contemplanti, i romiti, i solitari del deserto e della cella col corpo macero da' digiuni, da' cilizi e dalle vigilie, ritratti viventi de' misteri e delle 
leggende. C'erano gli umili di spirito, animati da schietto sentimento religioso e che tenevano la scienza come cosa profana, e ci erano i dotti, i predicatori e i confessori, il cui testo era la Bibbia e i santi Padri. Vedevi gli scolastici e gli eruditi, teologi e filosofi, che univano in una comune ammirazione i classici e i santi Padri, disputatori sottili di tutte le cose e anche delle cose di fede, parlanti un latino d'uso e di scuola, vibrato, rapido, vivace, dove sentivi il volgare destinato a succedergli, amici della filosofia con quello stesso ardore di fede che gli altri si professavano servi del Signore, ma di una filosofia non ripugnante alla fede, anzi sostegno, illustrazione e ragione di quella, confortata da sillogismi e da sentenze e da citazioni, dove trovi spesso Tullio accanto a san Paolo. Alteri della loro scienza e del loro latino, spregiatori del volgare, da costoro uscivano que' trattati, que' comenti, quelle "somme», quelle storie, che empivano di maraviglia il mondo. Accanto a questi veggenti della fede e della filosofia, a questa vita dello spirito, trovi la vita attiva e temporale, affratellati dallo stesso pensiero i signori e i tirannetti feudali e i priori e gli anziani delle repubbliche, il cavaliere de' romanzi e il mercatante delle cronache. Là appiè del Coliseo, un ardito negoziante, Giovanni Villani, pensò che la sua Fiorenza, figliuola di Roma, era non meno degna di avere una storia, e la scrisse. Fra tanto splendore e potenza del chiericato, lo spregiato laico cominciava a levare la testa e pensava all'antica Roma e a Firenze, figliuola di Roma. Là molte amicizie si strinsero, molte paci si fecero, come avviene in certi grandi momenti della storia umana; sparirono guelfi e ghibellini, ottimati e popolari, baroni e vassalli, stretti tutti ad una sola bandiera: uno Dio, uno papa, uno imperatore. Là il papato ebbe l'ultimo suo gran giorno, l'ultimo sogno di monarchia universale, rotto per sempre dallo schiaffo di Anagni. (SLI, p. 174-175)

Senti, in questa pagina, che l'autore vuol costruire, o ricostruire, proiettandolo nei secoli futuri, lo spirito (il Geist) della nazione e, con mossa tipicamente risorgimentale, si sforza di ritrovarne (o inventarne) le radici nella lontana civiltà comunale, religiosa e laica a un tempo, officiata da Mazzini e Gioberti. I concetti dominanti sono quelli che gli storici del Risorgimento a noi contemporanei hanno analizzato con grande cura: di nazione, di patria, di popolo. ${ }^{3}$

Le nazioni hanno, secondo De Sanctis, una missione e una vita:

Come gli individui hanno la loro missione in terra, così anche le nazioni. Gl'individui senza patria, senza virtù, senza gloria sono atomi perduti, «numerus fruges consumare nati». E parimenti ci sono nazioni oziose e vuote, che non lasciano alcun vestigio di sé nel mondo. [...] Ciò che rende grandi le nazioni è la virtù o la tempra, gagliardia intellettuale e corporale, che forma il carattere o la forza morale. Ma come gl'individui, così le nazioni hanno la loro vecchiezza, quando le idee che le hanno costituite s'indeboliscono nella coscienza e la tempra si fiacca. E l'indirizzo del mondo fugge loro dalle mani e passa ad altre nazioni. (SLI, p. 603)

3. Per esempio: Silvio LANARO, Patria: circumnavigazione di un'idea controversa, Venezia: Marsilio, 1996; Alberto Maria BANTI, La nazione nel risorgimento: parentela, santità e onore alle origini dell'Italia unita, Torino: Einaudi, 2006. 
La patria è anche più importante: essa va servita con passione e per essa si deve esser disposti a morire. Così, purtroppo, in Italia non è sempre stato. Qual è, per esempio, l'attaccamento alla patria di Giovanni Boccaccio?

Spento è in lui il cristiano, e anche il cittadino. Non gli è mai venuto in mente che servire la patria e dare a lei l'ingegno e le sostanze e la vita è un dovere così stretto, come è il provvedere al proprio sostentamento. Dietro al cittadino comincia a comparire il buon borghese, che ama la sua patria, ma a patto non gli dia molto fastidio, e lo lasci attendere alla sua industria, e non lo tiri per forza di casa o di bottega. (SLI, p. 363)

Sembra fare eccezione, staccandosi anche in questo, almeno in parte, Machiavelli:

Machiavelli, per la sua coltura letteraria, per la vita licenziosa, per lo spirito beffardo e motteggevole e comico, si lega al Boccaccio, a Lorenzo e a tutta la nuova letteratura. Non crede a nessuna religione, e perciò le accetta tutte, e magnificando la morale in astratto vi passa sopra nella pratica della vita. Ma ha l'animo fortemente temprato e rinvigorito negli uffici e nelle lotte politiche, aguzzato negli ozii ingrati e solitari. E la sua coscienza non è vuota. Ci è lì dentro la libertà e l'indipendenza della patria. (SLI, p. 593)

Nella ricerca del "genio» nazionale (specifico e distinto da quello degli «stranieri») e di un "sentimento politico elevato e nazionale», non resta che ritornare all'età comunale e mettere di nuovo, su un piedistallo, Dante Alighieri, il vero «italiano». Con non poca forzatura storica Dante, che era anzitutto fiorentino e sostenitore del potere sovranazionale dell'impero, diviene poeta risorgimentale:

A Dante non fa bisogno di rettorica. Si sente italiano e ne ha tutte le passioni, e ne senti il fremito e il tumulto nella sua poesia. (SLI, p. 339)

\section{La Storia della letteratura italiana come romanzo di formazione}

Fra le caratteristiche tipiche del romanzo di formazione De Sanctis ha fatto uso, anzitutto, del tema principale: quello che ricostruisce la crescita graduale, attraverso errori, correzioni e una progressiva presa di coscienza di sé, di un protagonista: in questo caso non un singolo individuo ma un intero popolo, o meglio «la coscienza del popolo italiano». Altre caratteristiche del genere a cui si è riallacciato sono: un impianto narrativo teleologico, verso la piena realizzazione del progetto di educazione o formazione; un forte contenuto etico-morale; una costante applicazione della metafora biologica della nascita, crescita e maturazione.

Di particolare interesse sono alcuni elementi della tessitura narrativa e dell'impianto linguistico del testo, che gli danno un'impronta chiaramente romanzesca, come il ricorso frequentissimo a nessi e legami narrativi che scandiscono l'evoluzione temporale della vita dei protagonisti. Sono comunissimi 
gli elementi di raccordo temporale del tipo «non ancora... ancora... non più... già... ecco». Ci sono poi parole e termini che vengono ripetuti e ribaditi con frequenza e rappresentano l'impianto concettuale della storia, segnandone il percorso attraverso lo schema storico dalla situazione originaria di freschezza civile, fervore vitale e severo impegno etico alla decadenza morale (accompagnata da un amore per la forma e la bellezza) alla «rigenerazione», alla «risurrezione», al «rinnovamento», al «risorgimento». A dare forza e plasticità a questo percorso provvedono molte metafore biologiche e naturalistiche, quali quelle del «nascere», del "prender carne», del «vivere», dell'essere «ancora in culla» e poi del «venire a maturità», della «sonnolenza», del «risveglio».

Ne do alcuni esempi:

[Nella poesia cortigiana] hai tutt'i difetti della decadenza, un seicentismo che infetta l'arte ancora in culla. (SLI, p. 71)

[Nelle Vite del Cavalca] il concetto del secolo, uscito dalle astrattezze teologiche e scolastiche, prende carne, acquista una esistenza morale e materiale. (SLI, p. 177)

[La Divina commedia] è una di quelle costruzioni gigantesche e primitive, vere enciclopedie, bibbie nazionali, non questo o quel genere, ma il tutto, che contiene nel suo grembo ancora involute tutta la materia e tutte le forme poetiche, il germe di ogni sviluppo ulteriore. (SLI, p. 243)

[Nel Canzoniere di Petrarca] molti generi di letteratura ancora iniziali e abbozzati già nella Commedia [vennero] a maturità. (SLI, p. 354)

[Abbandonati il gusto della lotta e messa a tacere la coscienza, nell'Italia del Seicento prevalse una generale sonnolenza e] il primo fenomeno di questa sonnolenza italiana fu il meccanismo, una stagnazione nelle idee, uno studio di fissare e immobilizzare le forme. Si arrestò ogni movimento filosofico e speculativo. (SLI, p. 678)

E potrei continuare a lungo con questa esemplificazione.

\section{La Storia della letteratura come dramma borghese}

Dal dramma borghese (e dal melodramma) derivano alcuni altri esempi, come per esempio il forte spicco che ricevono alcuni personaggi-protagonisti: Jacopone, Cavalcanti, Dante, Beatrice, Caterina da Siena, Petrarca, Boccaccio, Machiavelli, Ariosto, Folengo, Aretino, Sarpi, Tasso, Vico, Alfieri). Inoltre il gusto delle contrapposizioni dialettiche molto nette e potenzialmente drammatiche: Ciacco Dell'Anguillara e Ciullo d'Alcamo, Dante e Guido Cavalcanti, Dante e Petrarca, Dante e Ariosto, Dante e Vico, Boccaccio e Molière, Boccaccio e Voltaire, Machiavelli e Guicciardini, Galilei e Goldoni, Goldoni e Gozzi, ecc.

Infine la gustosa mescolanza di elementi drammatici e di elementi comici, per cui succede che Ludovico Ariosto, complici le Satire, di cui De Sanctis non 
avverte la dimensione letteraria e oraziana, diventa un personaggio da romanzo e viene messo in compagnia con Sancio Panza e Don Abbondio:

Il poveruomo era un personaggio idillico, non aveva ambizioni, non curava grandezze, né onori: «gli sapeva meglio una rapa in casa sua che tordo e starna all'altrui mensa» [...]. Ma non è lasciato vivere, e ha tra' piedi il cardinale, e ne sente una stizza che sfoga con questo e con quello. Qualche rara volta la stizza si alza a indignazione e gli strappa nobili accenti [...]. Ma sono scarse faville. Non è così rimesso d'animo o cupido d'onori, che imiti i cortigiani e sacrifichi la sua comodità per far a gusto del cardinale; e non è così altero che rompa la catena una buona volta, e lo mandi con Dio. Scrive borbottando e sfogando il mal umore, con una sua propria fisionomia nella scala de' Sancio Panza e de' don Abbondio. (SLI, p. 536-537)

L'avvio alla vita e alla carriera di molti personaggi è simile a quello di tanti protagonisti di romanzi borghesi:

[Ludovico Ariosto nel 1498] proprio l'anno che il Machiavelli era eletto segretario del comune fiorentino [...] aveva allora ventisei anni. Cinque ne aveva sciupati intorno alle leggi; finché, avuta dal padre licenza, si mise con ardore allo studio delle lettere, e tutto pieno il capo di Virgilio, Orazio, Petrarca, Plauto, Terenzio, cominciò a far versi latini e italiani, come tutti facevano. (SLI, p. 526-527)

Carlo Goldoni era, come Metastasio, artista nato. Di tutti e due se ne volea fare degli avvocati. Anzi Goldoni fece l'avvocato con qualche successo. Ma alla prima occasione correva appresso agli attori, insino a che il natural genio vinse. (SLI, p. 908)

[Giuseppe Parini]. Venuto dal contado in Milano, cominciò i soliti studii classici sotto i barnabiti, e il padre Branda fu suo maestro di rettorica. Il babbo volle farne un prete per nobilitare il casato; ma sul più bello fu costretto per le strettezze domestiche a troncare i suoi studii e a ingegnarsi per trarre innanzi la vita. (SLI, p. 922)

La metafora teatrale è molto frequente:

Il mondo [di Boccaccio] è il teatro de' fatti umani abbandonati al libero arbitrio e guidati ne' loro effetti dal caso. (SLI, p. 393)

Così come è frequente l'allusione al mascheramento e ai costumi degli attori:

[Lorenzo de' Medici] era il più fiorentino fra' fiorentini, non della vecchia stampa, s'intende. Cristiano e platonico in astratto e a scuola, in realtà epicureo e indifferente, sotto abito signorile popolano e mercante. (SLI, p. 444)

Frequenti sono le espressioni che chiamano in scena il lettore e gli suggeriscono come porsi di fronte ai vari personaggi: questo "ha l'aria di...", di quest'altro «senti in lui» uno o altro atteggiamento psicologico. Un tratto carat- 
teristico, che ha una forte intonazione drammatica e scultorea, lo si ha nell'uso del verbo "gittare», che già abbiamo incontrato nella descrizione del personaggio di Savonarola. Eccone un altro esempio, a proposito del personaggio dantesco di Vanni Fucci:

Ma l'umano non muore mai in tutto. L'uomo diviene bestia ${ }^{4}$, ma la bestia torna uomo. E con senso profondo Dante anche sulla faccia sfrontata di Vanni Fucci scoperto ladro gitta il rossore della vergogna. (SLI, p. 268)

\section{Strategie narrative}

Basta confrontare la Storia della letteratura italiana con altre due forme praticate da De Sanctis, e cioè la lezione universitaria e il saggio destinato alla pubblicazione in rivista, per cogliere la forte specificità narrativa della Storia.

Fra gli elementi che danno forza e compattezza narrativa a questo testo si possono annoverare: a) la rappresentazione della testualità letteraria come «spazio, come «mondo» che il narratore visita, invitando il lettore a visitarlo a sua volta; b) la tendenza alla personificazione della società nazionale, l'Italia, fornita di una coscienza e di uno spirito, trasformata in personaggio e anche alla personificazione delle epoche storiche. La tradizione letteraria italiana, attraverso una fitta rete di collegamenti interni, è rappresentata come un tutto continuo, con propri caratteri molto spiccati, all'interno della tradizione europea.

Quello della costruzione di uno «spazio» letterario italiano mi sembra una soluzione molto originale. Il narratore assume il tono della guida, del maestro, che porta con sé gli allievi e i lettori a visitare quello, come fosse un museo. Usa il «noi» del plurale. Non è un narratore onnisciente, ma certo un narratore che ha le sue buone e ampie conoscenze, ha davanti i suoi testi e senza farsene sopraffare li comunica, con svelta eleganza, agli ascoltatori e lettori:

C'incontriamo dapprima nella letteratura claustrale, ascetica, mistica, religiosa [...] Senti che quegli uomini prendono viva partecipazione a quello che scrivono, e vivono là dentro, e ci lasciano l'impronta del loro carattere e della loro fisionomia intellettuale e morale. Usciamo dalle astrattezze... (SLI, p. 176)

Entriamo in questo mondo [quello di Dante] e guardiamolo in sé stesso e interroghiamolo... (SLI, p. 239)

[In Petrarca troviamo] un doppio mondo [...] un mondo che se ne va e un mondo che se ne viene. (SLI, p. 348)

Mettiamo ora il piè in questo mondo del Boccaccio. Che vi troviamo? (SLI, p. 364)

Inchiniamoci innanzi a Giordano Bruno. (SLI, p. 766)

4. Questa espressione riecheggerà nel titolo di un famoso romanzo di Émile Zola: La bête bumaine (1890). 
La tendenza alle personificazioni la si incontra a ogni passo. Prima a essere personificata è l'Italia, come nelle immagini e nei monumenti del Risorgimento: la nazione con la sua storia, il suo passato, la sua «vita», il suo spirito che si incarna in tanti suoi figli:

In questa risurrezione dell'antica Italia è naturale che la lingua latina fosse stimata non solo lingua de' dotti, ma lingua nazionale, e che la storia di Roma dovesse sembrare agl'Italiani la loro propria storia. (SLI, p. 328)

L'Ariosto, il Machiavelli, l'Aretino sono le tre forme dello spirito italiano a quel tempo. (SLI, p. 672)

[Il Cinquecento:] quello era il tempo che l'Italia non solo non riusciva a fondare la patria, ma perdeva affatto la sua indipendenza, la sua libertà, il suo primato nella storia del mondo. Di questa catastrofe non ci era una coscienza nazionale, anzi ci era una certa soddisfazione. (SLI, p. 673)

Personificate sono le epoche storiche:

Il concetto del secolo [il Trecento], uscito dalle astrattezze teologiche e scolastiche, prende carne, acquista una esistenza morale e materiale. (SLI, p. 177)

[Boccaccio] volge le spalle al medio evo e inizia la letteratura moderna. (SLI, p. 379)

Intanto il secolo [il Settecento] camminava con passo sempre più celere. (SLI, p. 855)

Personificati sono i singoli testi, elevati a canone, divenuti monumento. Tali sono la Divina Commedia, il Principe, la Scienza nuova:

[La Scienza nuova di Vico] è la Divina commedia della scienza, la vasta sintesi, che riassume il passato e apre l'avvenire. (SLI, p. 850)

E così si stabilisce un ultimo grande nesso narrativo, un ulteriore elemento «legato» della trama. 